Pure and Applied Mathematics Quarterly

Volume 1, Number 2

(Special Issue: In memory of

Armand Borel, part 1 of 3)

$369-378,2005$

\title{
Arithmetic Mirror Symmetry
}

\author{
Daqing Wan
}

\begin{abstract}
This expository paper is based on my lecture at the Borel memorial conference. Our main purpose was to explore possible mirror relations between the arithmetic of a Calabi-Yau hypersurface and the arithmetic of its mirror. Through the study of some concrete examples, we discover that there are at least two types of mirror relations between the zeta function of a Calabi-Yau variety and the zeta function of its mirror. The aim of this note is to explain these relations.
\end{abstract}

\section{Mirror symmetry}

Definition 1.1 Let $n$ be a positive integer and let $K$ be a field. A smooth projective variety over $K$ of dimension $n$ is called Calabi-Yau (CY) if the following two conditions hold.

(i) The canonical bundle $\Omega_{X}^{n}$ is trivial.

(ii) The coherent sheaf cohomology $H^{i}\left(X, \mathcal{O}_{X}\right)$ vanishes for all $1 \leq i \leq n-1$.

In particular, a $\mathrm{CY}$ is connected. As an example, let $X$ be a smooth projective hypersurface in $\mathbb{P}^{n+1}$ of degree $d$ defined by the vanishing of a homogeneous polynomial $f\left(x_{0}, \cdots, x_{n+1}\right)$ of degree $d$. Then, $X$ is a Calabi-Yau hypersurface if and only if $d=n+2$. In the case $n=1$, this gives a cubic curve (elliptic curve). In the case $n=2$, this gives a quartic surface (K3-surface). In the case $n=3$, this gives a quintic 3 -fold (quintic CY).

Received April 5, 2005. 
Sometimes, $X$ has a mirror $X^{\circ}$ which is another smooth projective CY of dimension $n$. A basic question in mirror symmetry is to determine when a given pair $(X, Y)$ of $n$-dimensional $\mathrm{CY}$ varieties over $K$ forms a mirror pair. A necessary condition (the topological mirror test) for $X$ and $Y$ to be a mirror pair is that their Hodge numbers satisfy the Hodge symmetry:

$$
h^{i, j}(X)=h^{n-i, j}(Y), 0 \leq i, j \leq n .
$$

In particular, their Euler characteristics are related by

$$
e(X)=(-1)^{n} e(Y)
$$

In general, there is no known rigorous algebraic geometric definition for a mirror pair, although many examples of mirror pairs are known at least conjecturally. Furthermore, it does not make sense to speak of "the mirror" of $X$ as the mirror variety usually comes in a family. In some cases, the mirror does not exist. This is the case for rigid Calabi-Yau 3-folds $X$, since the rigid condition $h^{2,1}(X)=0$ would imply that $h^{1,1}(Y)=0$ which is impossible.

We shall assume that $X$ and $Y$ are a given mirror pair in some sense and are defined over a finite field. We are interested in how the zeta function of $X$ is related to the zeta function of $Y$. Since there is no algebraic geometric definition for $X$ and $Y$ to be a mirror pair, it is difficult to study the possible symmetry between their zeta functions in full generality. On the other hand, there are many explicit examples and constructions which at least conjecturally give a mirror pair, most notably in the toric hypersurface setting as constructed by Batyrev [1]. Thus, we shall first examine an explicit example and see what kind of relations can be proved for their zeta functions in this case. This would then suggest what to expect in general.

\section{An example of mirror pairs}

Let $n \geq 1$ be a positive integer. We consider the universal family of CalabiYau complex hypersurfaces of degree $n+2$ in the projective space $\mathbb{P}^{n+1}$. Its mirror family is a one-parameter family of toric hypersurfaces. To construct the mirror family, we consider the one-parameter subfamily $X_{\lambda}$ of complex projective hypersurfaces of degree $n+2$ in $\mathbb{P}^{n+1}$ defined by

$$
f\left(x_{0}, \cdots, x_{n+1}\right)=x_{0}^{n+2}+\cdots+x_{n+1}^{n+2}+\lambda x_{0} \cdots x_{n+1}=0,
$$

where $\lambda \in \mathbb{C}$ is the parameter. The variety $X_{\lambda}$ is a Calabi-Yau manifold when $X_{\lambda}$ is smooth. Let $\mu_{n+2}$ denote the group of $(n+2)$-th roots of unity. Let

$$
G=\left\{\left(\zeta_{0}, \cdots, \zeta_{n+1}\right) \mid \zeta_{i}^{n+2}=1, \zeta_{0} \cdots \zeta_{n+1}=1\right\} / \mu_{n+2} \cong(\mathbb{Z} /(n+2) \mathbb{Z})^{n},
$$


where $\mu_{n+2}$ is embedded in $G$ via the diagonal embedding. The finite group $G$ acts on $X_{\lambda}$ by

$$
\left(\zeta_{0}, \cdots, \zeta_{n+1}\right)\left(x_{0}, \cdots, x_{n+1}\right)=\left(\zeta_{0} x_{0}, \cdots, \zeta_{n+1} x_{n+1}\right) .
$$

The quotient $X_{\lambda} / G$ is a projective toric hypersurface $Y_{\lambda}$ in the toric variety $\mathbb{P}_{\Delta}$, where $\mathbb{P}_{\Delta}$ is the simplex in $\mathbb{R}^{n+1}$ with vertices $\left\{e_{1}, \cdots, e_{n+1},-\left(e_{1}+\cdots+e_{n+1}\right)\right\}$ and the $e_{i}$ 's are the standard coordinate vectors in $\mathbb{R}^{n+1}$. Explicitly, the variety $Y_{\lambda}$ is the projective closure in $\mathbb{P}_{\Delta}$ of the affine toric hypersurface in $\mathbb{G}_{m}^{n+1}$ defined by

$$
g\left(x_{1}, \cdots, x_{n+1}\right)=x_{1}+\cdots+x_{n+1}+\frac{1}{x_{1} \cdots x_{n+1}}+\lambda=0 .
$$

Assume that $X_{\lambda}$ is smooth. Then, $Y_{\lambda}$ is a (singular) mirror of $X_{\lambda}$. It is an orbifold. If $W_{\lambda}$ is a smooth crepant resolution of $Y_{\lambda}$, then the pair $\left(X_{\lambda}, W_{\lambda}\right)$ is called a mirror pair of Calabi-Yau manifolds. Such a resolution exists for this example but not unique if $n \geq 2$. In fact, let $\Delta^{*}$ be the dual polytope of $\Delta$. One checks that $\Delta^{*}$ is the simplex in $\mathbb{R}^{n+1}$ with the vertices

$$
(n+2) e_{i}-\sum_{j=1}^{n+1} e_{j}(i=1, \ldots, n+1),-\sum_{j=1}^{n+1} e_{j} .
$$

This is the $(n+2)$-multiple of a basic (regular) simplex in $\mathbb{R}^{n+1}$. In particular, the codimension 1 faces of $\Delta^{*}$ are $(n+2)$-multiples of a basic simplex in $\mathbb{R}^{n}$. By the parallel hyperplane decomposition in [4], one deduces that the codimension 1 faces of $\Delta^{*}$ have a triangulation into basic simplices. Any such a triangulation produces a smooth crepant resolution $\phi: W_{\lambda} \rightarrow Y_{\lambda}$.

The number of rational points and the zeta function are independent of the choice of the crepant resolution. We are interested in understanding how the arithmetic of $X_{\lambda}$ is related to the arithmetic of $W_{\lambda}$, in particular how the zeta function of $X_{\lambda}$ is related to the zeta function of $W_{\lambda}$. Our main concern in this paper is to consider Calabi-Yau manifolds over finite fields.

In this example, we see two types of mirror pairs. The first one is the generic mirror pair $\left\{X_{\Lambda}, W_{\lambda}\right\}$, where $X_{\Lambda}$ is the generic member in the moduli space of smooth projective Calabi-Yau hypersurfaces of degree $(n+2)$ in $\mathbb{P}^{n+1}$ and $W_{\lambda}$ is the generic member in the above one-parameter family of mirror Calabi-Yau manifolds. Note that $X_{\Lambda}$ and $Y_{\lambda}$ are parametrized by different parameter spaces (of different dimensions). The possible zeta symmetry in this case would then have to be a relation between certain generic property of their zeta functions. Later, we shall see that there is such a generic symmetry for the slope zeta function, which is a $p$-adic quantum version of the usual zeta function. 
The second type of mirror pairs is the one-parameter family of mirror pairs $\left\{X_{\lambda}, W_{\lambda}\right\}$ parametrized by the same parameter $\lambda$. This is a stronger type of mirror pair than the first type. For $\lambda \in \mathbb{C}$, we say that $W_{\lambda}$ is a strong mirror of $X_{\lambda}$. For such a strong mirror pair $\left\{X_{\lambda}, W_{\lambda}\right\}$, we can really ask for the relation between the zeta function of $X_{\lambda}$ and the zeta function of $W_{\lambda}$. We shall see that there is indeed a close relation between the zeta function of $X_{\lambda}$ and the zeta function of its strong mirror $W_{\lambda}$.

If $\lambda_{1} \neq \lambda_{2}, W_{\lambda_{1}}$ would not be called a strong mirror for $X_{\lambda_{2}}$, although they would be an usual weak mirror pair. In this case, we cannot expect a close relation between their zeta functions as the zeta function $Z\left(X_{\lambda}, T\right)$ depends in an essential way on the algebraic parameter $\lambda$.

Apparently, we do not have a definition for a strong mirror pair in general, as there is not even a definition for a generic or weak mirror pair in general.

\section{$3 \quad$ Arithmetic mirror symmetry}

Let $\mathbb{F}_{q}$ be a finite field of $q$ elements, where $q=p^{r}$ and $p$ is a prime. For a scheme $X$ of finite type of dimension $n$ over $\mathbb{F}_{q}$, let $\# X\left(\mathbb{F}_{q}\right)$ denote the number

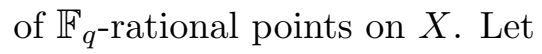

$$
Z(X, T)=\exp \left(\sum_{k=1}^{\infty} \frac{T^{k}}{k} \# X\left(\mathbb{F}_{q^{k}}\right)\right) \in 1+T \mathbb{Z}[[T]]
$$

be the zeta function of $X$. It is well known that $Z(X, T)$ is a rational function in $T$ whose reciprocal zeros and reciprocal poles are Weil $q$-integers. Factor $Z(X, T)$ over the $p$-adic numbers $\mathbb{C}_{p}$ and write

$$
Z(X, T)=\prod_{i}\left(1-\alpha_{i} T\right)^{ \pm 1}
$$

in reduced form, where the algebraic integers $\alpha_{i} \in \mathbb{C}_{p}$. One knows that the slope $\operatorname{ord}_{q}\left(\alpha_{i}\right)$ is a rational number in the interval $[0, n]$. For two real numbers $s_{1} \leq s_{2}$, we define the slope $\left[s_{1}, s_{2}\right]$ part of $Z(X, T)$ to be the partial product

$$
Z_{\left[s_{1}, s_{2}\right]}(X, T)=\prod_{s_{1} \leq \operatorname{ord}_{q}\left(\alpha_{i}\right) \leq s_{2}}\left(1-\alpha_{i} T\right)^{ \pm 1} .
$$

For a half open and half closed interval $\left[s_{1}, s_{2}\right), Z_{\left[s_{1}, s_{2}\right)}(X, T)$ is defined in a similar way. These are rational functions with coefficients in $\mathbb{Z}_{p}$ by the $p$-adic Weierstrass factorization. It is clear that we have the decomposition

$$
Z(X, T)=\prod_{i=0}^{n} Z_{[i, i+1)}(X, T) .
$$


Question 3.1 Let $\left(X, X^{\circ}\right)$ be a mirror pair of $C Y$ over $\mathbb{F}_{q}$.

(i) How is $\# X\left(\mathbb{F}_{q}\right)$ related to $\# X^{\circ}\left(\mathbb{F}_{q}\right)$ ?

(ii) How is $Z(X, T)$ related to $Z\left(X^{\circ}, T\right)$ ?

We propose

Conjecture 3.2 (Congruence mirror conjecture) Suppose that we are given a strong mirror pair $\left(X, X^{\circ}\right)$ of $C Y$ over $\mathbb{F}_{q}$.

(i) For each positive integer $k$, we have

$$
\# X\left(\mathbb{F}_{q^{k}}\right) \equiv \# X^{\circ}\left(\mathbb{F}_{q^{k}}\right)\left(\bmod q^{k}\right) .
$$

(ii) Equivalently, we have

$$
Z_{[0,1)}(X, T)=Z_{[0,1)}\left(X^{\circ}, T\right) .
$$

Part of the problem in the conjecture is that we do not know a general definition of strong mirror pairs. For the example in the previous section, we can indeed prove that the conjecture is true. This gives the following arithmetic mirror theorem.

Theorem 3.3 (Wan [9]) Assume that $\lambda \in \mathbb{F}_{q}$ such that $\left(X_{\lambda}, W_{\lambda}\right)$ is a strong mirror pair of Calabi-Yau manifolds over $\mathbb{F}_{q}$.

(i) For every positive integer $k$, we have the congruence formula

$$
\# X_{\lambda}\left(\mathbb{F}_{q^{k}}\right) \equiv \# Y_{\lambda}\left(\mathbb{F}_{q^{k}}\right) \equiv \# W_{\lambda}\left(\mathbb{F}_{q^{k}}\right)\left(\bmod q^{k}\right) .
$$

(ii) Equivalently, the slope $[0,1)$ part of the zeta function is the same for the mirror varieties $\left\{X_{\lambda}, Y_{\lambda}, W_{\lambda}\right\}$ :

$$
Z_{[0,1)}\left(X_{\lambda}, T\right)=Z_{[0,1)}\left(Y_{\lambda}, T\right)=Z_{[0,1)}\left(W_{\lambda}, T\right) .
$$

As an immediate corollary, we obtain

Corollary 3.4 Under the assumption of the previous theorem, there is a rational function $R_{n}(\lambda, T) \in 1+T \mathbb{Z}[[T]]$ which is pure of weight $n-2$ and degree $\frac{(n+1)\left((n+1)^{n+1}-(-1)^{n+1}\right)}{(n+2)}-(n+1)$ such that

$$
\left(\frac{Z\left(X_{\lambda}, T\right)}{Z\left(Y_{\lambda}, T\right)}\right)^{(-1)^{n+1}}=R_{n}(\lambda, q T)
$$


We conjecture that $R_{n}(\lambda, T)$ is actually a polynomial. This is known [9] to be true in the case that either $(n+2) \mid(q-1)$ or $(n+2, q-1)=1$. In particular, the integrality of $R_{n}(\lambda, T)$ is true if $n+2$ is a prime. In the case $n+2=5$, the polynomial $R_{3}(\lambda, T)$ with integer coefficients has degree 200. In [2], This degree 200 polynomial has empirically been shown to be the numerator of the zeta function of several genus 4 curves over $\mathbb{F}_{q}$.

The above mirror example was obtained via a quotient construction. More generally, in a joint work with Lei Fu, using the theory of crystalline cohomology and Mazur's result [6], we have proved the following partial generalization for the congruence mirror theorem.

Theorem 3.5 (Fu-Wan[3]) Let $X$ be a smooth Calabi-Yau scheme defined over the ring $W\left(\mathbb{F}_{q}\right)$ of Witt vectors of $\mathbb{F}_{q}$. Let $G$ be a finite group of $W\left(\mathbb{F}_{q}\right)$-morphisms acting on $X$. Assume that $G$ fixes the non-zero global section of the canonical bundle of $X$. Then, for each positive integer $k$, we have the congruence formula

$$
\#\left(X \otimes \mathbb{F}_{q}\right)\left(\mathbb{F}_{q^{k}}\right) \equiv \#\left(X / G \otimes \mathbb{F}_{q}\right)\left(\mathbb{F}_{q^{k}}\right)\left(\bmod q^{k}\right) .
$$

This theorem can also be proved using rigid cohomology and DeRham-Witt cohomology as shown by Esnault-Bloch.

\section{Slope zeta functions}

Assume that $\{X, Y\}$ forms a mirror pair, not necessarily a strong mirror pair. A different type of arithmetic mirror symmetry reflecting the Hodge symmetry, which is discrete and hence generic in nature, is to look for a suitable quantum version $Z_{Q}(X, T)$ of the zeta function such that

$$
Z_{Q}(X, T)=Z_{Q}(Y, T)^{(-1)^{n}}
$$

where $\{X, Y\}$ is a mirror pair of Calabi-Yau manifolds over $\mathbb{F}_{q}$ of dimension $n$. This relation cannot hold for the usual zeta function $Z(X, T)$ for obvious reasons, even for a strong mirror pair as it contradicts with the congruence mirror conjecture for odd $n$. No non-trivial candidate for $Z_{Q}(X, T)$ has been found. Here we propose a $p$-adic quantum version which would have the conjectural properties for most (and hence generic) mirror pairs. We will call our new zeta function to be the slope zeta function as it is based on the slopes of the zeros and poles.

Definition 4.1 For a scheme $X$ of finite type over $\mathbb{F}_{q}$, write as before

$$
Z(X, T)=\prod_{i}\left(1-\alpha_{i} T\right)^{ \pm 1}
$$


in reduced form, where $\alpha_{i} \in \mathbb{C}_{p}$. Define the slope zeta function of $X$ to be the two variable function

$$
S_{p}(X, u, T)=\prod_{i}\left(1-u^{\operatorname{ord}_{q}\left(\alpha_{i}\right)} T\right)^{ \pm 1} .
$$

Note that

$$
\alpha_{i}=q^{\operatorname{ord}_{q}\left(\alpha_{i}\right)} \beta_{i},
$$

where $\beta_{i}$ is a $p$-adic unit. Thus, the slope zeta function $S_{p}(X, u, T)$ is obtained from the $p$-adic factorization of $Z(X, T)$ by dropping the $p$-adic unit parts of the roots and replacing $q$ by the variable $u$. This is not always a rational function in $u$ and $T$. It is rational if all slopes are integers. Note that the definition of the slope zeta function is independent of the choice of the ground field $\mathbb{F}_{q}$ where $X$ is defined. It depends only on $X \otimes \overline{\mathbb{F}}_{q}$ and thus is also a geometric invariant. It would be interesting to see if there is a diophantine interpretation of the slope zeta function. If we have a smooth proper family of varieties, the Grothendieck specialization theorem implies that the generic Newton polygon on each cohomology exists and hence the generic slope zeta function exists as well.

The slope zeta function satisfies a functional equation [9]. This follows from the usual functional equation which in turn is a consequence of the Poincare duality for $\ell$-adic cohomology. Precisely, we have

Proposition 4.2 Let $X$ be a connected smooth projective variety of dimension $n$ over $\mathbb{F}_{q}$. Then the slope zeta function $S_{p}(X, u, T)$ satisfies the following functional equation

$$
S_{p}\left(X, u, \frac{1}{u^{n} T}\right)=S_{p}(X, u, T)\left(-u^{n / 2} T\right)^{e(X)},
$$

where $e(X)$ denotes the the $\ell$-adic Euler characteristic of $X$.

Suppose that $X$ and $Y$ form a mirror pair of $n$-dimensional Calabi-Yau manifolds over $\mathbb{F}_{q}$. For simplicity and for comparison with Hodge theory, we always assume in this paper that $X$ and $Y$ can be lifted to characteristic zero (to the Witt ring of $\mathbb{F}_{q}$ ). In this good reduction case, the modulo $p$ Hodge numbers equal the characteristic zero Hodge numbers. Taking $u=1$ in the definition of the slope zeta function, we see that the specialization $S_{p}(X, 1, T)$ already satisfies the desired relation

$$
S_{p}(X, 1, T)=(1-T)^{-e(X)}=(1-T)^{-(-1)^{n} e(Y)}=S_{p}(Y, 1, T)^{(-1)^{n}} .
$$

This suggests that there is a chance that the slope zeta function might satisfy the desired slope mirror symmetry

$$
S_{p}(X, u, T)=S_{p}(Y, u, T)^{(-1)^{n}} .
$$


If $n \leq 2$, the congruence mirror conjecture implies that the slope zeta function does satisfy the expected slope mirror symmetry for a strong mirror pair $\{X, Y\}$, whether $X$ and $Y$ are ordinary or not. For $n \geq 3$, we believe that the slope zeta function is still a little bit too strong for the expected symmetry to hold in general, even if $\{X, Y\}$ forms a strong mirror pair. And it should not be too hard to find a counter-example although we have not done so. However, we believe that the expected slope mirror symmetry holds for a generic mirror pair of 3-dimensional Calabi-Yau manifolds.

Conjecture 4.3 (Slope mirror conjecture) Suppose that we are given a generic mirror pair $\{X, Y\}$ of 3-dimensional Calabi-Yau manifolds defined over $\mathbb{F}_{q}$. Then, we have the slope mirror symmetry for their generic slope zeta functions:

$$
S_{p}(X, u, T)=\frac{1}{S_{p}(Y, u, T)} .
$$

A main point of this conjecture is that it holds for all prime numbers $p$. For arbitrary $n \geq 4$, the corresponding slope mirror conjecture might be false for some prime numbers $p$, but it should be true for all primes $p \equiv 1(\bmod D)$ for some positive integer $D$ depending on the mirror family, if the family comes from the reduction modulo $p$ of a family defined over a number field. In the case $n \leq 3$, one could take $D=1$ and hence get the above conjecture.

Again the condition in the slope mirror conjecture is vague as it is not presently known an algebraic geometric definition of a mirror family, although many examples are known in the toric setting. In a future paper, using the results in [7][8], we shall prove that the slope mirror conjecture holds in the toric hypersurface case if $n \leq 3$. For example, if $X$ is a generic quintic hypersurface, then $X$ is ordinary by the results in [5][7] for every $p$ and thus one finds

$$
S_{p}\left(X \otimes \mathbb{F}_{p}, u, T\right)=\frac{(1-T)(1-u T)^{101}\left(1-u^{2} T\right)^{101}\left(1-u^{3} T\right)}{(1-T)(1-u T)\left(1-u^{2} T\right)\left(1-u^{3} T\right)} .
$$

This is independent of $p$. Note that we do not know if the one-parameter subfamily $X_{\lambda}$ is generically ordinary for every $p$. The ordinary property for every $p$ was established only for the universal family of hypersurfaces, not for a one-parameter subfamily of hypersurfaces such as $X_{\lambda}$. If $Y$ denotes the generic mirror of $X$, then by the results in [7][8], $Y$ is ordinary for every $p$ and thus we obtain

$$
S_{p}\left(Y \otimes \mathbb{F}_{p}, u, T\right)=\frac{(1-T)(1-u T)\left(1-u^{2} T\right)\left(1-u^{3} T\right)}{(1-T)(1-u T)^{101}\left(1-u^{2} T\right)^{101}\left(1-u^{3} T\right)} .
$$

Again, it is independent of $p$. The slope mirror conjecture holds in this example. 
Remark: The slope zeta function is completely determined by the Newton polygon of the Frobenius acting on cohomologies of the variety in question. The converse is not true, as there may be cancellations coming from different cohomology dimensions in the slope zeta function.

From now on, we assume that $X$ is a smooth projective scheme over $W\left(\mathbb{F}_{q}\right)$. Assume that the reduction $X \otimes \mathbb{F}_{q}$ is ordinary, i.e., the $p$-adic Newton polygon coincides with the Hodge polygon [6]. In this case, one gets the explicit formula

$$
S_{p}\left(X \otimes \mathbb{F}_{q}, u, T\right)=\prod_{j=0}^{n}\left(1-u^{j} T\right)^{e_{j}(X)},
$$

where

$$
e_{j}(X)=(-1)^{j} \sum_{i=0}^{n}(-1)^{i-1} h^{j, i}(X)
$$

If $X$ and $Y$ form a mirror pair over the Witt ring $W\left(\mathbb{F}_{q}\right)$, the Hodge symmetry $h^{j, i}(X)=h^{j, n-i}(Y)$ implies for each $j$,

$$
e_{j}(X)=(-1)^{j} \sum_{i=0}^{n}(-1)^{i-1} h^{j, n-i}(Y)=(-1)^{n} e_{j}(Y) .
$$

We obtain the following result.

Proposition 4.4 Let $X$ and $Y$ be a mirror pair of n-dimensional smooth projective Calabi-Yau schemes over $W\left(\mathbb{F}_{q}\right)$. Assume that both $X \otimes \mathbb{F}_{q}$ and $Y \otimes \mathbb{F}_{q}$ are ordinary. Then, we have the following symmetry for the slope zeta function:

$$
S_{p}\left(X \otimes \mathbb{F}_{q}, u, T\right)=S_{p}\left(Y \otimes \mathbb{F}_{q}, u, T\right)^{(-1)^{n}} .
$$

The converse of this proposition may not be always true. The slope mirror conjecture follows from the following slightly stronger

Conjecture 4.5 (Generically ordinary conjecture) Let $n \leq 3$. Suppose that $\{X, Y\}$ form a generic mirror pair of $n$-dimensional smooth projective Calabi-Yau schemes over $W\left(\mathbb{F}_{q}\right)$. Then, both $X \otimes \mathbb{F}_{q}$ and $Y \otimes \mathbb{F}_{q}$ are generically ordinary.

For $n \leq 3$, it is possible to prove this conjecture in the toric hypersurface case using the results in[7][8]. For $n \geq 4$, we expect that the same conjecture holds if $p \equiv 1(\bmod D)$ for some positive integer $D$. This should again be provable in the toric hypersurface case using the results in [7]. But we do not know if we can always take $D=1$, even in the toric hypersurface case if $n \geq 4$. 


\section{References}

[1] V. Batyrev, Dual polyhedra and mirror symmetry for Calabi-Yau hypersurfaces in toric varieties, J. Algebraic Geom., 3(1994), no. 3, 493-535.

[2] P. Candelas, X. de la Ossa and F. Rodriguez-Villegas, Calabi-Yau manifolds over finite fields, II, preprint, 2004. "arXiv:hep-th/0402133".

[3] L. Fu and D. Wan, Mirror congruence for rational points on Calabi-Yau varieties, arXiv:math.AG/0503703, 30 March 2005.

[4] G. Kempf, F. Knudsen, D. Mumford and B. Saint-Donat, Toroidal Embeddings, Lecture Notes in Math., 339, Spriner-Verlag, 1973.

[5] L. Illusie, Ordinarité des intersections complètes générales, in Grothendieck Festschrift, Volume II (1990), 375-405.

[6] B. Mazur, Frobenius and the Hodge filtration, Bull. Amer. Math. Soc., 78(1972), 653-667.

[7] D. Wan, Newton polygons of zeta functions and L-functions, Ann. Math., 137(1993), 247-293.

[8] D. Wan, Variation of $p$-adic Newton polygons for L-functions of exponential sums, Asian J. Math., Vol. 8, 3(2004), 427-474.

[9] D. Wan, Mirror symmetry for zeta functions, arXiv:math.AG/0411464, 22 November 2004.

Daqing Wan

Institute of Mathematics

Chinese Academy of Sciences

Beijing, P.R. China

and

Department of Mathematics

University of California

Irvine, CA 92697-3875

E-mail:dwan@math.uci.edu 